\title{
Evaluation of Correlation between Clinical and Magnetic Resonance Findings of Patellar Chondromalacia
}

\author{
Deniz Özel ${ }^{1}$, ๑ Mustafa Çağlar Kır² ${ }^{2}$, Mehmet Öncü ${ }^{3}$
}

${ }^{1}$ Department of Radiology, University of Health Sciences Turkey, Okmeydani Health Aplication and Research Center, Istanbul, Turkey

${ }^{2}$ Department of Orthopedics, University of Health Sciences Turkey, Okmeydani Health Aplication and Research Center, Istanbul, Turkey

${ }^{3}$ Department of Radiology, University of Health Sciences, İstanbul Bağcılar Training and Research Hospital, Istanbul, Turkey

\begin{abstract}
Introduction: To evaluate the correlation between clinical appearance and magnetic resonance imaging features of chondromalacia that can determine the knee magnetic resonance indication and to define a novel chondral status indicator. Methods: This study was performed between May 2017 and February 2019. If the knee pain was suggestive for the presence of chondromalacia clinically, then VAS and Kujala AKPS were noted. For MRI evaluation, a patellar cartilage score (PCS) was defined to evaluate the mean patellar thickness (MPT) and MRI grading together.

Results: Advanced chondromalacia was more common among females $(p<0.01)$. There was a positive correlation between age and MRI grading and the correlation coefficient was calculated to be 0.56 . The highest correlation coefficient obtained with PCS in comparing PCS, MCT (mean cartilage thickness) and MRI grading for the clinical relationship. To define advanced chondromalacia with VAS and AKPS cut-off values calculated to be 3 and 80 in ROC analysis. AKPS was a superior clinical indicator for advanced chondromalacia compared with VAS. The other demographic findings, such as age and gender relations, were concordant with the literature.

Discussion and Conclusion: A potentially better method was defined to combine VAS with functional measurements, such as AKPS, to determine a knee status may be indicative of MRI evaluation. As a novel perspective, PCS was the best indicator of the chondral clinical status that consists of both the mean patellar thickness and magnetic resonance grading.

Keywords: Clinical correlation; MRl; patellar chondromalacia.
\end{abstract}

B esides being a common issue, chondromalacia is an expected result of aging. Beginning in childhood, cartilaginous tissues becomes progressively thinner and degenerated (such as the presence of fissures, fragmentation and tissue loss) ${ }^{[1,2]}$. Clinical evaluation of chondromalacia is being performed with many methods, but the two of these methods named visual analogue score (VAS) and Kujala anterior knee pain scale (AKPS) are often applied. VAS is a common and well-known physical 10 scores examination method. On the other hand, as a functional clinical test, AKPS is a more specific, self-report questionnaire that consists of thirteen items, especially used for sports medicine and orthopaedics. The scoring system documents response to the activities associated with anterior knee pain syndrome, and symptoms, such as stiffness, inability to weight-bearing, swelling, abnormal patellar movement, 
muscular atrophy and limited flexion of the knee. This test asks about the duration of complaints. The highest possible score is one hundred and lower ratings indicate greater pain/disability ${ }^{[3,4]}$.

For confusing cases, additional knee MRI is performed to confirm the diagnosis. According to the literature, the two main methods of patellar chondromalacia can be identified with conventional knee MRI. The first method is defining and grading the patellar cartilage signal intensity and the second method is measuring the mean thickness of the cartilaginous tissue.

This study aimed to evaluate the correlation between clinical appearance and MRI features of chondromalacia and to define a novel MRI indicator to diagnose patellar chondromalacia. After a search of the literature, we discovered that many articles are drawing the usefulness of new MRI techniques and their correlations with arthroscopy. However, there are few articles about the correlation between clinical appearance and commonly used MRI features, so we hoped to contribute to the existing knowledge ${ }^{[5,6]}$.

\section{Materials and Methods}

We prospectively reviewed the clinical scores of the patients who underwent the knee MRI and diagnosed to be chondromalacia based on clinical scores. The knee MRI images Chondromalacia staging was performed by the same radiologist with a 4-year experience. This study was performed in our institute between May 2017 and February 2019. If the knee pain was suggestive for the presence of chondromalacia clinically, then VAS and Kujala AKPS were noted. Corresponding MR features were evaluated. If any individual had additional knee disturbance/s, those could be a reason for the knee pain, such as meniscal or ligament injuries, inflammatory processes or masses; they excluded from this study to objectively determine the source of the pain-disturbance. Another reason for exclusion was the presence of motion artifacts that prevent optimal cartilage evaluation. The patients with pediatric age were also excluded from this study because of ongoing maturation, so the patellar cartilage thickness and the signal intensity could be confusing.

\section{MRI Technique}

All examinations were performed using a 1.5 Tesla magnet (Signa Excite, GE Medical Systems, Waukesha, Wisconsin, USA). A compatible knee coil was used. All patients underwent axial, coronal and sagittal proton density-weighted and coronal T1 weighted imaging. Slice thickness was 4-5 $\mathrm{mm}$ without the intersection gap.
On MRI examination, the mean patellar cartilage thickness (MCT) and MRI grading were performed for each patient. Modified Outerbridge classification was used for MRI grading. This is the five-score (between 0 and 4) system defining cartilage status ${ }^{[7]}$.

The MCT was calculated to be the arithmetic mean of four measurements of the patellar posterolateral cartilage tissue thickness (thinnest and thickest medial component and thinnest and thickest lateral component) on proton density-weighted axial images (Fig. 1). MRI evaluations performed by the same musculoskeletal radiologist.

\section{Defining the Patellar Cartilage Score}

From the very beginning of our study, we aimed to define a parameter that we can evaluatethe mean thickness of the patellar cartilage and the MRI grade together that can be a novel indicator. For this purpose, patellar cartilage score (PCS) was defined to be: MCT $(\mathrm{mm}) /(\mathrm{MRI}$ grade +1$)$. Here, the presence of +1 was to avoid division by zero for Modified Outerbridge grade 0 cartilages.

\section{Statistical Analysis}

Categorical variables were expressed as percentages. Comparison of chondromalacia grading ratios via patient genders analyzed using Pearson's chi-square test. Pearson's correlation test and linear regression analysis were used for comparing MPT, MRI grade and PCS for chondral status indicators. The ROC analysis, positive (PPV) and negative predictive values (NPV), diagnostic accuracy value (DAV) were calculated to compare VAS and AKPS. A p-value of equal to or less than 0.05 was considered as significant. Microsoft Excel program version 1805 was used for data storage and statistical calculations.

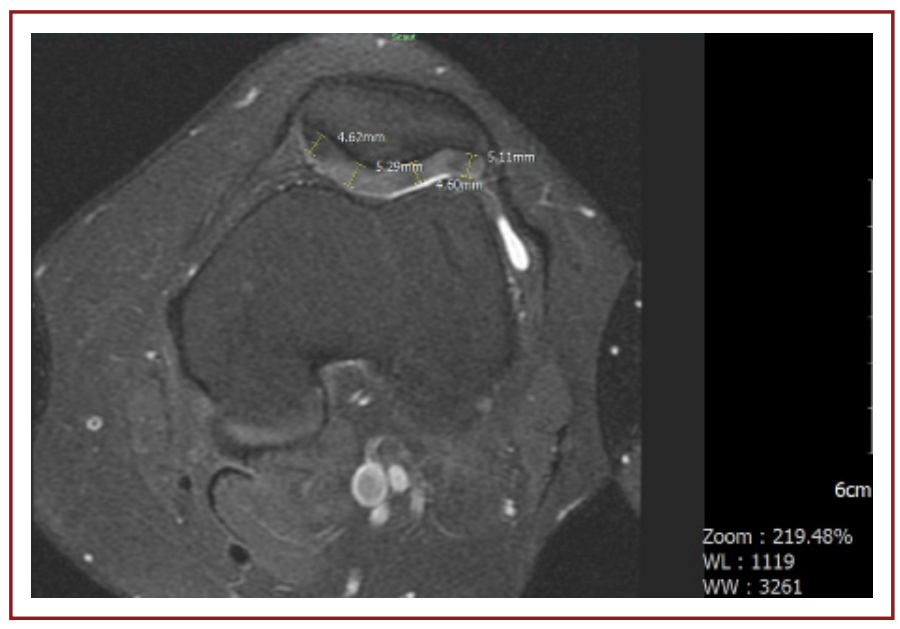

Figure 1. Calculating the mean patellar cartilage thickness. 


\section{Results}

This study included 67 patients. Forty-four of the patients (65.7\%) were female, and 23 (34.3\%) were male. The mean age of the patients was 40.13 , ranging from 19 to 67 years.

Advanced (grade 3 and 4) chondromalacia was seen more common among females $(p<0.01)$. There was a positive correlation between the age and the MRI grade, and correlation coefficient was calculated to be 0.56 (Table 1 ).

In the comparison of chondral status parameters, PCS had the highest correlation coefficient. The differences between correlation coefficients were statistically significant in the regression analysis (Table 2 ).

After PCS defined to be the best cartilage status indicator, then an advanced disease group was identified. This group included the patients with the lowest 20 PCS indicators. All MRI grade 3 and 4 patients were placed in this group with the additional two grade 2 patients. In the comparison of VAS and AKPS, the cut-off values calculated to be 3 and 80, respectively in ROC analysis. PPV, NPV and DAV were slightly higher for AKPS (Table 3).

Table 1. Demographic data according to the MRI grade for chondromalacia

\begin{tabular}{lcccc}
\hline & Mean age \pm SD & Males & Females & Female/Male ratio \\
\hline Grade 0 & $31.5 \pm 9.3$ & 8 & 9 & 1.13 \\
Grade 1 & $38.7 \pm 11$ & 9 & 15 & 1.67 \\
Grade 2 & $43.1 \pm 6.3$ & 4 & 4 & 1 \\
Grade 3 & $44 \pm 8$ & 1 & 6 & 6 \\
Grade 4 & $51.3 \pm 9$ & 1 & 10 & 10 \\
& $\mathrm{r}=0.56$ & Total: 23 & Total: 44 & Mean: 1.82 \\
\hline
\end{tabular}

Table 2. Pearson correlation coefficients for the clinical scores and MRI parameters

\begin{tabular}{lccc}
\hline & MCT & MRI grade & PCS \\
\hline VAS & -0.381 & 0.391 & -0.514 \\
AKPS & 0.481 & -0.552 & 0.637 \\
Age & -0.53 & 0.56 & -0.57 \\
\hline
\end{tabular}

Table 3. Evaluation of the VAS and AKPS scores for advanced chondromalacia patients

\begin{tabular}{lccc}
\hline Cut off values & PPV & NPV & Diagnostic accuracy \\
\hline AKPS $=80$ & 0.33 & 0.93 & 0.66 \\
VAS $=3$ & 0.26 & 0.87 & 0.63 \\
\hline
\end{tabular}

Figures 2 and 3 show MRl images of the two patients placed on the opposite side of the cartilage status scale.

Figure 4 shows AKPS superiority when compared with VAS, presents cartilage status much closer.

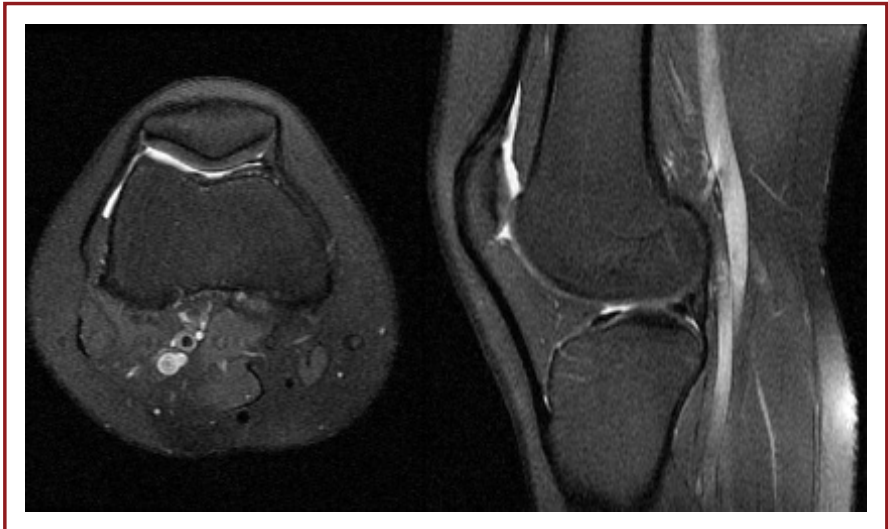

Figure 2. 19 years old male. Patellar cartilage score: 3.8 VAS: 2 AKPS: 91 (MRI Grade-0).

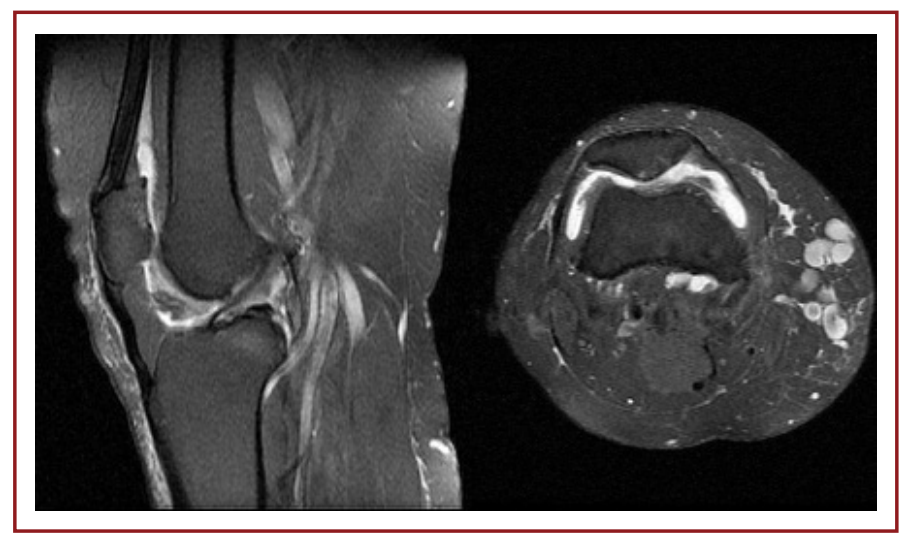

Figure 3. 60 years old female. Patellar cartilage score: 0.4 VAS: 7 AKPS: 62 (MRI Grade-4).

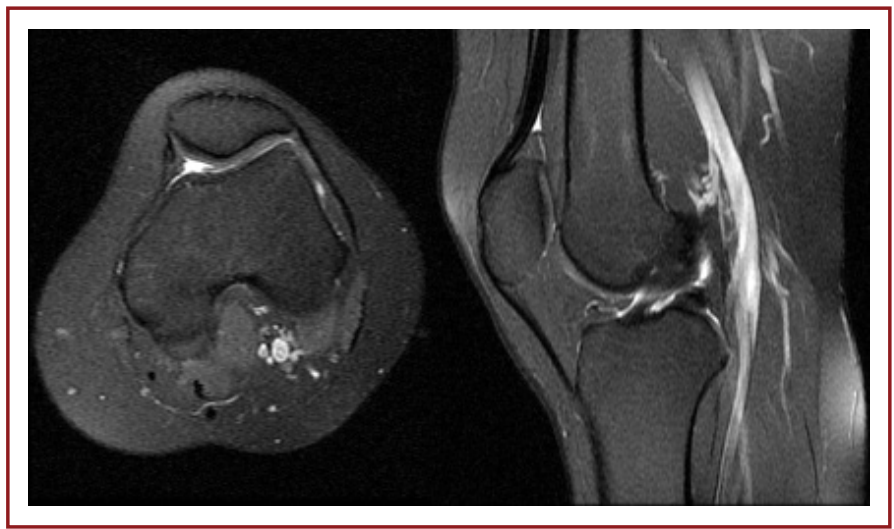

Figure 4. 29 years old female. Patellar cartilage score: 3.4 VAS: 4 AKPS: 87. In this case, AKPS presents cartilage status much closer compared with VAS. 


\section{Discussion}

According to our results, AKPS was a superior advanced chondromalacia indicator when compared with VAS. This result can be expected since it is a specific test for the knee and has dynamic components measuring activities additionally. However, despite its widespread acceptance clinically, relatively few studies have reported on its technical properties with pediatric patients. Since none of the individuals included in this study was younger than 18 years old, this situation was not an issue for our study. We also never experienced a single advanced chondromalacia patient younger than eighteen, which was not expressed in the results section. This result shows that although $\mathrm{CP}$ is a pain-centered condition, it is a better idea to combine VAS with functional measurements, such as APKS.

In knee MRI evaluation, MPT was higher in male individuals. Thinner cartilage tissue may cause clinical findings earlier because any structural defect constitutes a larger percentage of the cartilage tissue. In addition, subchondral degenerative changes, including bone marrow edema, tend to be seen earlier. According to our results, chondromalacia shows female predominance, especially for highgrade (advanced) disease, which makes sense. Sullivan et al.'s ${ }^{[8]}$ study also showed that the mean cartilage thickness was greater among men in various sites around the knee. Farrokhi et al. ${ }^{[9]}$ showed that the mean cartilage thickness was decreased in patients with patellofemoral pain. They also remarked that a cartilage thickness change was smaller after exercise. AKPS was their clinical method to define knee pain.

The patellar chondromalacia is not associated only with aging, but also there are environmental factors that may affect cartilage deformity. For instance, Wei et al. ${ }^{[10]}$ concluded that parity status was negatively associated with chondral degeneration, especially in multiparous women. This finding may be another reason for female predominance. They also found that body mass index was affected with parity, as a borderline factor ( $p$ was 0.05 ). There are also diseases, such as rheumatoid arthritis that may accelerate chondral degeneration and loss ${ }^{[11]}$. There was a positive correlation between the age and the MRI grade and a negative correlation between the age and MPT. As a result, there was a negative correlation between the age and PCS. The correlation coefficients were found to be close to each other. These results were consistent with the results from similar studies.

PCS was found to be the best correlated MRI indicator with the clinical findings. Since this novel score based on MRI grading and the mean cartilage thickness together, we could expect the highest correlation naturally. Keeping in mind that this method is easily applicable, radiologists can report that score to define the actual chondral status. In similar studies, chondral status is defined with different parameters. For example, Endo et al. ${ }^{[12]}$ reported, there was not a statistically significant correlation between patellar chondromalacia and different measurements of patellar facet length and the inter-facet angle. Conversely, Thakkar et al. ${ }^{[13]}$ and Chan et al. ${ }^{[14]}$ concluded quantitative trochlear parameters could be reliably calculated on MRI. The literature on quantitative imaging is usually related to the patellar malalignment ${ }^{[15-18]}$.

We must remind that there are several studies report that MRI cannot be labeled to be the best modality especially for low-graded patellar chondromalacia. The considerable limitation of this study was the lack of gold standard. Further studies combined with arthroscopy or MRI arthroscopy may offer more accurate results. Although conventional MRI has disadvantages in the diagnosis of low-grade chondral lesions, it still appears to be the most common method because arthroscopic techniques are invasive and have potential complications. In a similar study, indirect MRI arthrography had higher diagnostic accuracy, and its components than conventional MRI in knee pathologies, as well as arthrography. The patellar chondromalacia was one of those knee pathologies ${ }^{[19]}$. There are also articles report, MRI imaging is accepted as an accurate imaging method of detecting and grading moderate to advanced cartilage defects [20, 21].

There were two studies pointing closest to our results. Aysin IK et al. ${ }^{[5]}$ used AKPS as a clinical parameter, but their results were not comparable with our results since they used the different MRI parameters. Pihlajämaki et al. ${ }^{[6]}$ concluded patellar chondromalacia could not be diagnosed according to symptoms or with a current physical examination. However, the conclusion was based on level 1 lesions because of low sensitivity on $1 \mathrm{~T}$ MRI findings (with 13\% sensitivity). On the other hand, for the other grades of the disease, their results were satisfying. According to our results, AKPS had better diagnostic value, but on the other side of the coin, PPV values of VAS and AKPS were clearly low and reducing their diagnostic value.

As a summary, the diagnosis and grading of the low-graded chondral lesions with conventional MRI seems to be problematic. Since our clinical score comparison was based on high-graded chondromalacia, it was not an issue that conventional MRI's weaknesses on low graded chondral 
lesions; however, we must accept as the second limitation that, low graded chondral parameters were obtained for cartilage status indicators.

\section{Conclusion}

It is a better idea to combine VAS with functional measurements, such as APKS, which is being used to determine the knee MRI indication to evaluate chondromalacia clinically. For MRI evaluation, a novel perspective, patellar cartilage score was the best indicator that reflects chondral clinical status consisting of both the mean patellar thickness and the MRI grade, and this method is easily applicable.

Ethical Committee Approval: All procedures performed in studies involving human participants were in accordance with the ethical standards and an appropriate institutional committee approved them (Health Sciences University Okmeydanı SUAM Date 5.3.2019/No.1146).

Peer-review: Externally peer-reviewed.

Authorship Contributions: Concept: D.Ö., M.Ç.K., M.Ö.; Design: D.Ö, M.Ç.K., M.Ö.; Data Collection or Processing: D.Ö., M.Ç.K., M.Ö.; Analysis or Interpretation: D.Ö., M.Ç.K., M.Ö.; Literature Search: D.Ö., M.Ç.K., M.Ö.; Writing: D.Ö.

Conflict of Interest: None declared.

Financial Disclosure: The authors declared that this study received no financial support.

\section{References}

1. Kim HK, Shiraj S, Anton CG, Horn PS, Dardzinski BJ. Age and sex dependency of cartilage T2 relaxation time mapping in MRI of children and adolescents. AJR Am J Roentgenol 2014;202:626-32. [CrossRef]

2. Hudelmaier M, Glaser C, Hohe J, Englmeier $\mathrm{KH}$, Reiser M, Putz $\mathrm{R}$, et al. Age-related changes in the morphology and deformational behavior of knee joint cartilage. Arthritis Rheum 2001;44:2556-61. [CrossRef]

3. Kujala UM, Jaakkola LH, Koskinen SK, Taimela S, Hurme M, Nelimarkka O. Scoring of patellofemoral disorders. Arthroscopy 1993;9:159-63. [CrossRef]

4. Singer B, Singer K. Anterior Knee Pain Scale. Aust J Physiother 2009;55:140. [CrossRef]

5. Aysin IK, Askin A, Mete BD, Guvendi E, Aysin M, Kocyigit H. Investigation of the Relationship between Anterior Knee Pain and Chondromalacia Patellae and Patellofemoral Malalignment. Eurasian J Med 2018;50:28-33. [CrossRef]

6. Pihlajamäki HK, Kuikka PI, Leppänen VV, Kiuru MJ, Mattila VM. Reliability of clinical findings and magnetic resonance imaging for the diagnosis of chondromalacia patellae. J Bone Joint Surg Am 2010;92:927-34. [CrossRef]

7. Cole BJ, Malek MM. Articular cartilage lesions. New York: Springer, c2004. [CrossRef]
8. Sullivan NP, Robinson PW, Ansari A, Hassaballa M, Robinson $J R$, Porteous AJ, et al. Bristol index of patellar width to thickness (BIPWiT): a reproducible measure of patellar thickness from adult MRI. Knee 2014;21:1058-62. [CrossRef]

9. Farrokhi S, Colletti PM, Powers CM. Differences in patellar cartilage thickness, transverse relaxation time, and deformational behavior: a comparison of young women with and without patellofemoral pain. Am J Sports Med 2011;39:384-91. [CrossRef]

10. Wei S, Jones G, Venn A, Cicuttini F, March L, Otahal P, et al. The association between parity and knee cartilage in young women. Rheumatology (Oxford) 2012;51:2039-45. [CrossRef]

11. Gandy SJ, Brett AD, Dieppe PA, Keen MC, Maciewicz RA, Taylor $\mathrm{CJ}$, et al. Measurement of cartilage volumes in rheumatoid arthritis using MRI. Br J Radiol. 2005;78:39-45. [CrossRef]

12. Endo Y, Schweitzer ME, Bordalo-Rodrigues M, Rokito AS, Babb JS. MRI quantitative morphologic analysis of patellofemoral region: lack of correlation with chondromalacia patellae at surgery. AJR Am J Roentgenol 2007;189:1165-8. [CrossRef]

13. Thakkar RS, Del Grande F, Wadhwa V, Chalian M, Andreisek G, Carrino JA, et al. Patellar instability: CT and MRI measurements and their correlation with internal derangement findings. Knee Surg Sports Traumatol Arthrosc 2016;24:3021-8.

14. Chan VO, Moran DE, Mwangi I, Eustace SJ. Prevalence and clinical significance of chondromalacia isolated to the anterior margin of the lateral femoral condyle as a component of patellofemoral disease: observations at MR imaging. Skeletal Radiol 2013;42:1127-33. [CrossRef]

15. Stefanik JJ, Zumwalt AC, Segal NA, Lynch JA, Powers CM. Association between measures of patella height, morphologic features of the trochlea, and patellofemoral joint alignment: the MOST study. Clin Orthop Relat Res 2013;471:2641-8.

16. Narkbunnam R, Chareancholvanich K. Effect of patient position on measurement of patellar height ratio. Arch Orthop Trauma Surg 2015;135:1151-6. [CrossRef]

17. Mehl J, Feucht MJ, Bode G, Dovi-Akue D, Südkamp NP, Niemeyer P. Association between patellar cartilage defects and patellofemoral geometry: a matched-pair MRI comparison of patients with and without isolated patellar cartilage defects. Knee Surg Sports Traumatol Arthrosc 2016;24:838-46. [CrossRef]

18. Yue RA, Arendt EA, Tompkins MA. Patellar Height Measurements on Radiograph and Magnetic Resonance Imaging in Patellar Instability and Control Patients. J Knee Surg 2017;30:943-50. [CrossRef]

19. Boegård TL, Rudling O, Petersson IF, Jonsson K. Distribution of MR-detected cartilage defects of the patellofemoral joint in chronic knee pain. Osteoarthritis Cartilage 2003;11:494-8.

20. Murphy BJ. Evaluation of grades 3 and 4 chondromalacia of the knee using $\mathrm{T}^{*}{ }^{*}$-weighted $3 \mathrm{D}$ gradient-echo articular cartilage imaging. Skeletal Radiol 2001;30:305-11. [CrossRef]

21. Mathieu L, Bouchard A, Marchaland JP, Potet J, Fraboulet B, Danguy-des-Deserts $M$, et al. Knee MR-arthrography in assessment of meniscal and chondral lesions. Orthop Traumatol Surg Res 2009;95:40-7. [CrossRef] 